\title{
Adaptation of the de Hoffmann-Teller frame for quasi-perpendicular collisionless shocks
}

\author{
H. Comişel ${ }^{1,2}$, Y. Narita ${ }^{3,4}$, and U. Motschmann ${ }^{1,5}$ \\ ${ }^{1}$ Institut für Theoretische Physik, Technische Universität Braunschweig, Mendelssohnstr. 3, 38016 Brunswick, Germany \\ ${ }^{2}$ Institute for Space Sciences, Atomiştilor 409, P.O. Box MG-23, Bucharest-Măgurele 077125, Romania \\ ${ }^{3}$ Space Research Institute, Austrian Academy of Sciences, Schmiedlstr. 6, 8042 Graz, Austria \\ ${ }^{4}$ Institut für Geophysik und extraterrestrische Physik, Technische Universität Braunschweig, Mendelssohnstr. 3, \\ 38106 Brunswick, Germany \\ ${ }^{5}$ Deutsches Zentrum für Luft- und Raumfahrt, Institut für Planetenforschung, Rutherfordstr. 2, 12489 Berlin, Germany \\ Correspondence to: H. Comişel (h.comisel@tu-bs.de)
}

Received: 5 December 2014 - Revised: 26 February 2015 - Accepted: 27 February 2015 - Published: 17 March 2015

\begin{abstract}
The concept of the de Hoffmann-Teller frame is revisited for a high Mach-number quasi-perpendicular collisionless shock wave. Particle-in-cell simulation shows that the local magnetic field oscillations in the shock layer introduce a residual motional electric field in the de HoffmannTeller frame, which is misleading in that one may interpret that electrons were not accelerated but decelerated in the shock layer. We propose the concept of the adaptive de Hoffmann-Teller (AHT) frame in which the residual convective field is canceled by modulating the sliding velocity of the de Hoffmann-Teller frame. The electrostatic potential evaluated by Liouville mapping supports the potential profile obtained by electric field in this adaptive frame, offering a wide variety of applications in shock wave studies.
\end{abstract}

Keywords. Space plasma physics (shock waves)

\section{Introduction}

Understanding collisionless shock waves remains one of the challenges in space and astrophysical plasmas. The shock dissipation mechanism converts the kinetic energy of the incoming flow partly into thermal energy and partly into the energy of supra-thermal particles (Thomsen et al., 1987). There are different approaches to explain electron acceleration and heating processes in the collisionless shocks: (1) through the large-scale quasi-stationary electrostatic potential (called hereafter the cross-shock potential) and (2) through turbulent heating of the shock ramp (Scudder et al., 1986; Hull et al., 1998, 2000, 2001; Walker et al., 2004; Dimmock et al., 2011, 2012; Wilson III et al., 2014a). In this study, we limit the study to the understanding the heating process in a quasi-static electrostatic field using a numerical simulation in order to obtain a stationary picture of collisionless shock. Of course, fluctuating electromagnetic and electrostatic waves may contribute to heating (Breneman et al., 2013; Hull et al., 2006; Pulupa et al., 2010; Wilson III et al., 2007, 2010, 2012, 2014a, b), which is beyond the scope of our current study.

The Liouville theorem formulating the phase-space density conservation has successfully been applied to map the electron velocity distribution function in the upstream region onto that in the shock transition layer and further in the downstream region. This procedure, referred to as $\mathrm{Li}^{-}$ ouville mapping, provides the cross-shock potential that can explain the origin of the thermal and supra-thermal populations of electrons (e.g., Scudder et al., 1986; Scudder, 1995). The cross-shock potential is subject to the choice of the frame (Goodrich and Scudder, 1984). For example, it can be evaluated in the normal incident frame (NIF) in which the flow is aligned with the shock normal direction or in the de Hoffmann-Teller (HT) frame in which the flow is aligned with the upstream magnetic field on the both upstream and downstream sides. These two frames are related to each other by the Galilean transform using a constant frame velocity tangential to the shock front (called the sliding velocity). The utility of the de Hoffmann-Teller frame lies in that the motional (or convective) electric field is canceled such that one can study the electric field that arises from the electrostatic 
potential without being confused by the motional field. Furthermore, the use of the de Hoffmann-Teller frame can naturally be extended to the entire shock region so long as the electron flow velocity and the local magnetic field remain nearly parallel to each other within the shock transition layer (Scudder, 1987).

On the other hand, the collisionless shock is known to become more dynamic at a sufficiently high Mach number in that the structure of the transition layer becomes nonstationary, exhibiting various kinds of wave-particle interactions. If strong gradients occur in electric and magnetic fields at small scales in the shock front, one may expect that adiabatic heating of electrons can no longer function. For example, See et al. (2013) have shown that such short-scale largeamplitude structures of the electric field can switch the adiabatic heating regime into a non-adiabatic one, in accordance with previous theoretical studies of Balikhin et al. (1993), and in situ measurements, e.g., Walker et al. (2004) and Bale and Mozer (2007).

The demagnetization of electrons in the shock ramp is expected also for small-scale large-amplitude oscillations of the magnetic field direction. Furthermore, the de HoffmannTeller frame is no longer able to provide the electric field unique to the shock transition layer, since both the residual component of the motional electric field and the electrostatic field are measured simultaneously. In other words, the application of the constant sliding velocity for the de HoffmannTeller frame is no more valid to study the electric field nature of the shock transition layer properly. The breakdown of the validity of the de Hoffmann-Teller frame weakens the accurate measurement of the electric field for the cross-shock potential. However, the method of Liouville mapping has successfully been applied to determine the shock potential that is responsible for the electron heating. A question arises naturally: in which reference frame can we construct the shock potential profile using the electric field data?

Here, we present a numerical simulation study of the nonstationary shock. We obtain the answer that the motional electric field needs to be locally canceled within the shock transition layer by modulating the sliding velocity for the de Hoffmann-Teller frame. By doing so, it is possible to measure the electrostatic field and the shock potential for the electron heating properly as Liouville mapping does. We refer to the modulated frame as the Adaptive de HoffmannTeller frame (AHT). Without this correction, one may be misled to the conclusion that the electric field was acting to decelerate incoming electrons due to the dominance of residual component of the motional field in the de HoffmannTeller frame. We perform the one-dimensional particle-incell (PIC) simulation of a high Mach number, low-beta, quasi-perpendicular collisionless shock. Earlier PIC simulations have already shown that the shock wave becomes highly non-stationary under such a condition with various kinds of instabilities developing in the shock foot and the ramp regions. We track the spatial evolution of the electric field, the magnetic field, and the electrons through the shock when its transition is the steepest, and obtain the shock potential in two different ways: from the electric field measurement in the AHT frame and from Liouville mapping.

\section{Particle-in-cell simulation}

The shock wave is produced numerically using the "em1D" code (Birdsall and Langdon, 1991; Scholer et al., 2003a, b). Using this code, an electron-proton plasma is injected in the one-dimensional simulation box from the left-hand side. The plasma streams toward the right-hand side (the positive $x$ direction). The simulation box is set under a uniform magnetic field at an angle of $81^{\circ}$ from the shock normal (which points in the negative $x$ direction). The upstream magnetic field has two components, $B_{x}$ and $B_{z}$. At the boundary on the righthand side of the simulation box, the ions and the electrons are reflected by the "wall". The shock wave is formed and propagates in the negative $x$ direction. After a sufficiently long time, the shock wave reaches the boundary on the left-hand side and the whole simulation box becomes the downstream region. The shock wave in the simulation box is related to that in NIF in that the shock wave is not at rest but propagates in the negative $x$ direction.

The simulation box consists of a mesh with 40000 cells. Each cell has an equal size, the Debye length $\lambda_{\mathrm{D}}$. Time, length, and particle velocity are normalized to the inverse proton cyclotron frequency $\left(\Omega_{\mathrm{i}}\right)$, the electron inertial length $\left(\lambda_{\mathrm{e}}\right)$, and the speed of light in vacuum $(c)$, respectively. The magnetic field and electron density are scaled to their respective upstream values. The electrostatic potential is given in units of the product of the upstream magnetic field $B_{0}$ and the Debye length. Unless noted, we use the Gaussian units elsewhere. Five hundred particles for electrons and ions are set in each cell. Ions are assumed to be protons, but the ionto-electron mass ratio is set to 1000 for efficient computation. The upstream plasma flow obtains Alfvén Mach number 8. When the shock wave develops, the Alfvén Mach number reaches a value of about 10 . The value of plasma beta is 0.2 in the both species with a Maxwellian initial incident particle velocity distribution. The ratio of the electron plasma frequency to the electron gyrofrequency is 8 . Note that the values of beta and the frequency ratio are constrained to the maximum computational load, and not set to reproducing the collisionless shock in space such as Earth's bow shock (which is by far too demanding compared to the computation capacity available to date). During the simulation run, the shock wave forms and propagates, while it is highly nonstationary in that the shock transition layer exhibits the reformation process.

Figure 1 displays the snapshot (or the spatial profile) of the magnetic field (the $z$ or tangential component to the shock surface), the electron density, and the electron phase-space density at the time about 4.5 ion gyroperiods. No smooth- 

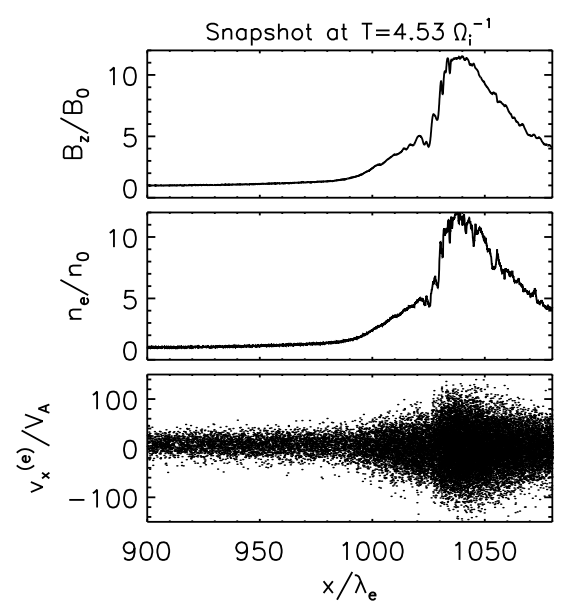

Figure 1. Snapshot of the magnetic field (the $z$ component), the electron density, and the subset of electron phase-space density at the time around 4.5 ion gyroperiods. The magnetic field and the electron density are normalized to the respective background values.

ing is applied here. It is interesting to note that the wave activity is the smallest in the maximum phase of the reformation shock in our simulation. Highly oblique shocks may exhibit turbulent ramp regions if the detectors have high enough time resolution in the spacecraft observations (Horbury et al., 2001; Hull et al., 2006; Lobzin et al., 2007; Wilson III et al., 2012, 2014a, b). The shock re-formation reaches its maximum and the transition layer is the narrowest with the largest transition amplitude in the magnetic field data. The transition is clearest and sharpest from upstream to downstream in the magnetic field and the density profile at this time. Wave activity is present throughout the foot region $\left(x \simeq 1000 \lambda_{\mathrm{e}}\right)$, the ramp region $\left(x \simeq 1020 \lambda_{\mathrm{e}}\right)$, and the overshoot region $\left(x \simeq 1040 \lambda_{\mathrm{e}}\right)$. In contrast, at the time one halfcycle earlier in re-formation (at the time about 3.5 ion gyroperiods), the foot region is extended over a larger spatial scales with higher wave amplitudes.

\section{Cross-shock potential}

The cross-shock potential is evaluated in two different ways: first by integrating the electric field in the adaptive de Hoffmann-Teller frame and second by Liouville mapping. The electric field in the adaptive de Hoffmann-Teller frame is constructed as follows.

$\boldsymbol{E}^{(\mathrm{AHT})}=\boldsymbol{E}^{(\mathrm{NIF})}+\boldsymbol{U} \times \boldsymbol{B}+\delta \boldsymbol{U} \times \boldsymbol{B}+\boldsymbol{U} \times \delta \boldsymbol{B}$,

where the symbol $\boldsymbol{E}^{(\mathrm{NIF})}$ denotes the electric field in the NIF frame obtained by correcting for the shock propagating speed, $\boldsymbol{U}$ and $\delta \boldsymbol{U}$ are the sliding velocity for the de Hoffmann-Teller frame and its modulation for the adaptive frame, and $\boldsymbol{B}$ and $\delta \boldsymbol{B}$ are the asymptotic upstream magnetic field (far from the shock transition) and the spatial oscilla- tion of the magnetic field within the transition layer. To obtain the electric field in the adaptive frame, the sliding velocity is modulated in the third term on the right-hand side to compensate for the residual motional electric field originating in the magnetic field fluctuation (the fourth term on the right-hand side). The electric field is obtained first in the NIF frame $\boldsymbol{E}^{(\mathrm{NIF})}$, and then transformed into the de HoffmannTeller frame $\boldsymbol{E}^{(\mathrm{HT})}$ (using the first and the second terms) and the adaptive de Hoffmann-Teller frame $\boldsymbol{E}^{(\mathrm{AHT})}$ (including the third and the fourth terms). We set the direction of the shock normal to the negative $x$ axis in our one-dimensional shock simulation in the adaptive de Hoffmann-Teller frame, and the local residual motional electric field is compensated with respect to the flow in the shock normal direction. It is worth mentioning that local compensation for the motional electric field in three dimensions is also possible.

The shock potential is obtained by the integration of the electric field over the spatial coordinate along the shock normal in the de Hoffmann-Teller frame $\left(\Phi^{(\mathrm{HT})}\right)$ and the adaptive frame $\left(\Phi^{(\mathrm{AHT})}\right)$. The potential is set to zero in the limit of far upstream. Figure 2 displays the electrostatic potentials $\Phi^{(\mathrm{HT})}$ and $\Phi^{(\mathrm{AHT})}$ as a function of the spatial coordinate around the shock transition layer at the time of shock re-formation maximum (4.5 ion gyroperiods after the simulation kickoff). Here, we applied smoothing in the HT and AHT potential profiles because of numerical noise. The $x$ axis range in Fig. 2 is extended to the border of simulation box (the wall). The electric potential should ideally be close to zero far from the shock but remains finite because of the relatively small box setup. It is interesting to note that the potentials have different signs. The potential has mostly negative values in the de Hoffmann-Teller frame. This is the effect of the residual component of the motional electric field (the third term in the equation) which originates in the spatially oscillating magnetic field. The representation of the potential in the de Hoffmann-Teller frame is misleading, since one might interpret it to mean that the shock potential was decelerating electrons. On the other hand, when the correction is undertaken for the oscillating magnetic field (by adding the third and the fourth terms), the shock potential is represented with the positive sign and the association of the potential with the electron acceleration is justified.

We evaluate the shock potential using the Liouville mapping of the electron distribution function at various distances from the upstream region (the simulation border on the lefthand side) to the shock transition layer. Liouville mapping is an alternative procedure to find the electrostatic potential by fitting the two distribution functions using the least square method on the assumption that the magnetic moments of individual particles are conserved. Frame transformation into AHT system was not applied to Liouville mapping. We used Liouville mapping (Lefebvre et al., 2007) in the de Hoffmann-Teller frame as a reference potential because Liouville mapping is a coordinate-free method. Figure 3 displays an example of the fitting procedure in Liou- 


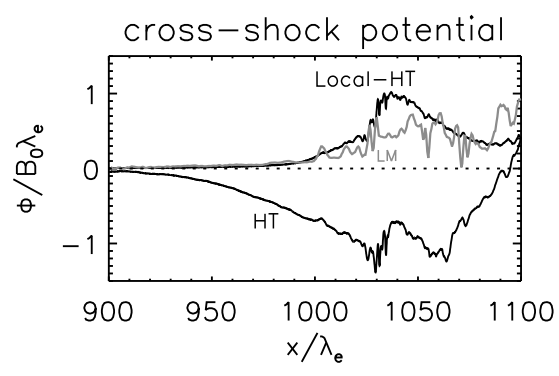

Figure 2. Cross-shock potential obtained by the three different methods: electric field integration in the global de Hoffmann Teller frame (solid curve in black in the lower panel); electric field integration in the adaptive de Hoffmann Teller frame (solid curve in black in the upper panel); and electrostatic potential obtained by Liouville mapping (in gray).

ville mapping at the time 4.5 ion gyroperiods and the spatial coordinate $x=1020 \lambda_{\mathrm{e}}$ and for the pitch angle $65^{\circ}$. The electron distribution function obtained by the PIC simulation is shown by the solid curve in black, and that obtained by Liouville mapping is in dotted symbols in gray. The profile of the cross-shock potential is obtained by Liouville mapping along the normal direction from the upstream region to the shock. This potential is over-plotted in Fig. 2 in gray. While the maximum potential value is different from the potential in the adaptive de Hoffmann-Teller frame, the sign and the asymptotic behavior of the potential agreements between $\mathrm{Li}$ ouville mapping and the adaptive frame.

The distribution function in Fig. 3 is obtained in the de Hoffmann-Teller frame for accelerated and heated electrons at the shock ramp. The sliding velocity $v_{\mathrm{dHT}}$ of this frame is rather high $\left(v_{\mathrm{dHt}} \simeq 0.2 c\right)$, since the shock has a high Mach number and a large angle from the upstream magnetic field. Incident particles with higher perpendicular energies are most likely reflected by the magnetic mirroring at the shock ramp, while particles with lower perpendicular energies can be trapped in the potential well of the existing overshoot (Hull et al., 2001).

Additionally, the validity of Liouville mapping is examined by comparing the distributions functions using two different methods: one is the direct measurement by counting the number of particles with various velocities, and the other one is the exact mapping (without assuming energy conservation nor magnetic moment conservation) of the phase-space density associated with the individual tracked particles. We find that the two distribution functions agree with each other for nearly adiabatic particles within the accuracy of magnetic moment conservation by greater than $90 \%$ between the initial and final stage of the mapping.

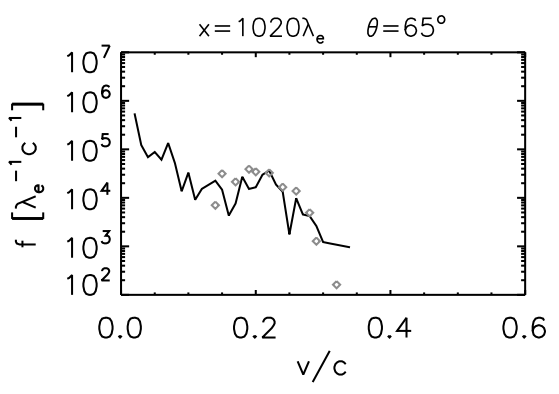

Figure 3. Example of the electron distribution function at the time shown in Fig. 1 in the de Hoffmann-Teller frame, the spatial coordinate $x=1020 \lambda_{\mathrm{e}}$ (at the shock ramp), and the pitch angle $65^{\circ} \mathrm{ob}-$ tained by the PIC simulation (solid line in black) and that obtained by Liouville mapping (in gray diamonds).

\section{Summary and discussion}

In the case of stationary shock, one may safely construct the de Hoffmann Teller frame and apply the method of Liouville mapping to determine the cross-shock potential. In the case of non-stationary shock, the existence and the uniqueness of the de Hoffmann Teller frame are no longer guaranteed. Nevertheless, the use of Liouville mapping is a valid approach. We find in this study that the construction of an adequate reference frame (the adaptive de Hoffmann-Teller frame) is possible for the study of electric field to validate the potential obtained by Liouville mapping by modulating the sliding velocity and correcting locally for the spatially oscillating magnetic field. Without this correction, one obtains the cross-shock potential in the de Hoffmann-Teller frame with the opposite sign, and may be misled to the conclusion that the shock potential were not accelerating but decelerating the electrons.

See et al. (2013) have proved that short-scale largeamplitude electric field structures within the electric field profile lead to incoherent heating of the electrons. In our study, we observe large-amplitude oscillations of the magnetic field direction that result in a residual motional electric field in the de Hoffmann-Teller frame. The local demagnetization of the adiabatic electrons should alter the quality of Liouville mapping, but overall, the electron thermalization remains primarily controlled by the coherent heating.

In the first-order picture, the high-frequency electrostatic waves should not influence the determination of the de Hoffmann-Teller frame because the impact of waves on the electromagnetic component $\boldsymbol{v}_{\mathrm{e}} \times \boldsymbol{B}$ is small, see e.g., Breneman et al. (2013), Mozer and Sundkvist (2013), and Wilson III et al. (2014a, b). In the second-order picture, however, there might be a possibility that the high-frequency electric field oscillation affects the electron bulk motion.

The cross-shock potential was analyzed using in situ measurements, e.g., in the NIF frame, by Walker et al. (2004), Dimmock et al. (2011, 2012), and Wilson III et al. (2014a), 
but determination of the cross-shock potential from direct spacecraft observations is still far too inaccurate in the de Hoffmann-Teller frame because the electric field is too small.

Various methods have been alternatively devised to evaluate the cross-shock potential (Hull et al., 2001): evaluation using the electron fluid momentum equation, that using the electron fluid energy equation, that using the electron polytrope assumption, and that using Liouville mapping.

These procedures are based on specific assumptions that are mutually dependent. Moreover, the irreversibility problem is not yet solved. Above all, we find that Liouville mapping is robust in that the method can be applied even to a non-stationary shock. In this way, the time evolution of the shock potential can be tracked throughout the shock reformation process. However, Liouville mapping relies on the assumption that the electrons are adiabatic, and the validity of this assumption needs to be examined by other means. In the adaptive de Hoffmann-Teller frame, the electric field can directly be associated with the electrostatic potential. This frame would be a convenient choice in order to track the evolution of the electron distribution function and that of the electric field through the shock wave.

Acknowledgements. This work was financially supported by ECSTRA/PECS and TUNED/STAR of the Romanian Space Agency under contract 11/2012, Collaborative Research Center 963 Astrophysical Flows, Instabilities and Turbulence by the German Science Foundation. This work was also supported by the Austria-Romania bilateral project ULF-MAG (Austrian Agency for International Cooperation in Education and Research, Centre for International Cooperation \& Mobility under contract RO12/2014 and 741/2014). The research leading to these results has received funding from the European Community's Seventh Framework Programme under the grant agreement 313038/STORM.

Topical Editor L. Blomberg thanks one anonymous referee for their help in evaluating this paper.

\section{References}

Bale, S. D. and Mozer, F. S.: Measurement of large parallel and perpendicular electric fields on electron spatial scales in the terrestrial bow shock, Phys. Rev. Lett., 98, 205001, doi:10.1103/PhysRevLett.98.205001, 2007.

Balikhin, M., Gedalin, M., and Petrukovich, A.: New mechanism for electron heating in shocks, Phys. Rev. Lett., 70, 1259-1262, doi:10.1103/PhysRevLett.70.1259, 1993.

Birdsall, C. K. and Langdon, A. B.: Plasma physics via computer simulations, IOP Publishing Ltd, 1991.

Breneman, A., Cattell, C., Kersten, K., Paradise, A., Schreiner, S., Kellogg, P. J., Goetz, K., and Wilson III, L. B.: STEREO and Wind observations of intense cyclotron harmonic waves at the Earth's bow shock and inside the magnetosheath, J. Geophys. Res., 118, 7654-7664, doi:10.1002/2013JA019372, 2013.
Dimmock, A. P., Balikhin, M. A., and Hobara, Y.: Comparison of three methods for the estimation of cross-shock electric potential using Cluster data, Ann. Geophys., 29, 815-822, doi:10.5194/angeo-29-815-2011, 2011.

Dimmock, A. P., Balikhin, M. A., Krasnoselskikh, V. V., Walker, S. N., Bale, S. D., and Hobara, Y.: A statistical study of the cross-shock electric potential at low Mach number, quasiperpendicular bow shock crossings using Cluster data, J. Geophys. Res., 117, A02210, doi:10.1029/2011JA017089, 2012.

Goodrich, C. C. and Scudder, J. D.: The adiabatic energy change of plasma electrons and the frame dependence of the cross-shock potential at collisionless magnetosonic shock waves, J. Geophys. Res., 89, 6654-6662, doi:10.1029/JA089iA08p06654, 1984.

Horbury, T. S., Cargill, P. J., Lucek, E. A., Balogh, A., Dunlop, M. W., Oddy, T. M., Carr, C., Brown, P., Szabo, A., and Fornaçon, K.-H.: Cluster magnetic field observations of the bowshock: Orientation, motion and structure, Ann. Geophys., 19, 1399-1409, doi:10.5194/angeo-19-1399-2001, 2001.

Hull, A. J., Scudder, J. D., Frank, L. A., Paterson, W. R., and Kivelson, M. G.: Electron heating and phase space signatures at strong and weak quasi-perpendicular shocks, J. Geophys. Res., 103, 2041-2054, doi:10.1029/97JA03058, 1998.

Hull, A. J., Scudder, J. D., Fitzenreiter, R. J., Ogilvie, K. W., Newbury, J. A., and Russell, C. T.: Electron temperature and de Hoffmann-Teller potential change across the Earth's bow shock: New results from ISEE 1, J. Geophys. Res., 105, 20957-20972, doi:10.1029/2000JA900049, 2000.

Hull, A. J., Scudder, J. D., Larson, D. E., and Lin, R.: Electron heating and phase space signatures at supercritical, fast mode shocks, J. Geophys. Res., 106, 15711-15734, doi:10.1029/2001JA900001, 2001.

Hull, A. J., Larson, D. E., Wilber, M., Scudder, J. D., Mozer, F. S., Russell, C. T., and Bale, S. D.: Large-amplitude electrostatic waves associated with magnetic ramp substructure at Earth's bow shock, Geophys. Res. Lett., 33, L15104, doi:10.1029/2005GL025564, 2006.

Lefebvre, B., Schwartz, S. J., Fazakerly, A. F., and Décréau, P.: Electron dynamics and cross-shock potential at the quasiperpendicular Earth's bow shock, J. Geophys. Res., 112, A09212, doi:10.1029/2007JA012277, 2007.

Lobzin, V. V., Krasnoselskikh, V. V., Bosqued, J., Pinçon, J., Schwartz, S. J., and Dunlop, M.: Nonstationarity and reformation of high-Mach-number quasiperpendicular shocks: Cluster observations, Geophys. Res. Lett., 34, L05107, doi:10.1029/2006GL029095, 2007.

Mozer, F. S. and Sundkvist, D.: Electron demagnetization and heating in quasi-perpendicular shocks, J. Geophys. Res., 118, 54155420, doi:10.1002/jgra.50534, 2013.

Pulupa, M. P., Bale, S. D., and Kasper, J. C.: Langmuir waves upstream of interplanetary shocks: Dependence on shock and plasma parameters, J. Geophys. Res., 115, A04106, doi:10.1029/2009JA014680, 2010.

Scholer, M., Shinohara, I., and Matsukiyo, S.: Quasi-perpendicular shocks:Length scale of the cross-shock potential, shock reformation, and implication for shock surfing, J. Geophys. Res., 108, 4.1-4.11, doi:10.1029/2002JA009515, 2003a.

Scholer, M., Kucharek, H., and Shinohara, I.: Short large-amplitude magnetic structures and whistler wave precursors in a full- 
particle quasi-parallel shock simulation, J. Geophys. Res., 108, 3.1-3.7, doi:10.1029/2002JA009820, 2003b.

Scudder, J. D.: The Field-aligned approximation for electrons within layers possessing a normal mass flux: A corollary to the de Hoffmann-Teller theorem, J. Geophys. Res., 92, 13447-13455, doi:10.1029/JA092iA12p13447, 1987.

Scudder, J. D.: A review of the physics of electron heating at collisionless shocks, Adv. Space Res., 15, 181-223, doi:10.1016/0273-1177(94)00101-6, 1995.

Scudder, J. D., Aggson, T. L., Mangeney, A., Lacombe, C., and Harvey, C. C.: The resolved layer of a collisionless, high beta, super-critical, quasi-perpendicular shock wave: I. RankineHugoniot geometry, currents, and stationarity, J. Geophys. Res., 91, 11019-11052, doi:10.1029/JA091iA10p11019, 1986.

See, V., Cameron, R. F., and Schwartz, S. J.: Non-adiabatic electron behaviour due to short-scale electric field structures at collisionless shock waves, Ann. Geophys., 31, 639-646, doi:10.5194/angeo-31-639-2013, 2013.

Thomsen, M. F., Stansberry, J. A., Bame, S. J., Gosling, J. T., and Mellott, M. M.: Strong electron heating at the earth's bow shock, J. Geophys. Res., 92, 10119-10124, doi:10.1029/JA092iA09p10119, 1987.

Walker, S. N., Alleyne, H. St. C. K., Balikhin, M. A., André, M., and Horbury, T. S.: Electric field scales at quasi-perpendicular shocks, Ann. Geophys., 22, 2291-2300, doi:10.5194/angeo-222291-2004, 2004.
Wilson III, L. B., Cattell, C., Kellogg, P. J., Goetz, K., Kersten, K., Hanson, L., MacGregor, R., and Kasper, J. C.: Waves in interplanetary shocks: A wind/waves study, Phys. Rev. Lett., 99, 41101, doi:10.1103/PhysRevLett.99.041101, 2007.

Wilson III, L. B., Cattell, C. A., Kellogg, P. J., Goetz, K., Kersten, K., Kasper, J. C., Szabo, A., and Wilber, M.: Large-amplitude electrostatic waves observed at a supercritical interplanetary shock, J. Geophys. Res., 115, A12104, doi:10.1029/2010JA015332, 2010.

Wilson III, L. B., Koval, A., Szabo, A., Breneman, A., Cattell, C. A., Goetz, K., Kellogg, P. J., Kersten, K., Kasper, J. C., Maruca, B. A., and Pulupa, M.: Observations of electromagnetic whistler precursors at supercritical interplanetary shocks, Geophys. Res. Lett., 39, L08109, doi:10.1029/2012GL051581, 2012.

Wilson III, L. B., Sibeck, D. G., Breneman, A. W., Le Contel, O., Cully, C., Turner, D. L., Angelopoulos, V., and Malaspina, D. M.: Quantified energy dissipation rates in the terrestrial bow shock: 1. Analysis techniques and methodology, J. Geophys. Res., 119, 6455-6474, doi:10.1002/2014JA019929, 2014a.

Wilson III, L. B., Sibeck, D. G., Breneman, A. W., Le Contel, O., Cully, C., Turner, D. L., Angelopoulos, V., and Malaspina, D. M.: Quantified energy dissipation rates in the terrestrial bow shock: 2. Waves and dissipation, J. Geophys. Res., 119, 6475-6495, doi:10.1002/2014JA019929, 2014b. 